\title{
Eye Position Effects in Oculomotor Plasticity and Visual Localization
}

\author{
Eckart Zimmermann ${ }^{1,2}$ and Markus Lappe ${ }^{3}$ \\ ${ }^{1}$ Department of Psychology, Università degli Studi di Firenze, 50135 Florence, Italy, ${ }^{2}$ Department of Physiological Sciences, Università di Pisa, 56123 Pisa, \\ Italy, and ${ }^{3}$ Department of Psychology and Otto Creutzfeldt Center for Cognitive and Behavioral Neuroscience, University of Muenster, 48149 Muenster, \\ Germany
}

For visual localization to remain accurate across changes of gaze, a signal representing the position of the eye in the orbita is needed to code spatial locations in a reference frame that is independent of retinal displacements. Here we report evidence that the localization of visual objects in space is coded in an extraretinal reference frame. In human subjects, we used outward saccadic adaptation, which can be induced artificially by a systematic displacement of the saccade target. This form of oculomotor plasticity is accompanied by changes in spatial perception, thus highlighting the relevance of saccade metrics for visual localization. We tested the reference frame of outward adaptation for reactive and scanning saccades and visual localization. For scanning saccades, adaptation magnitude was drastically reduced at positions distant from the adapted eye position. Changes in visual localization showed a very similar modulation of eye position. These results suggest that scanning saccade adaptation is encoded in a nonretinotopic reference frame. Eye position effects for reactive saccade adaptation were smaller, and the induced mislocalization did not vary significantly between eye positions. The different modulation of reactive and scanning saccade adaptation supports the idea that oculomotor plasticity can occur at multiple sites in the brain. The findings are also consistent with previous evidence for a stronger influence of scanning saccade adaptation on the visual localization of objects in space.

\section{Introduction}

To keep sensorimotor coordination accurate, the brain must compensate for the permanent changes of the retinal image resulting from changes in eye, head, and body position. Visual and motor targeting is directed toward locations in space, but gaze position is changed with every saccadic eye movement, on average three times per second. To stabilize spatial localization, retinal information must be combined with knowledge about the current position of the eye. Saccade targeting can be adaptively adjusted by artificially displacing the saccade target while the eye moves (McLaughlin, 1967; Hopp and Fuchs, 2004; Pélisson et al., 2010). Oculomotor plasticity is paralleled by changes in visual localization that shift the apparent position of visual objects in space (Bahcall and Kowler, 1999; Awater et al., 2005; Bruno and Morrone, 2007; Collins et al., 2007). Saccadic adaptationinduced distortions of visual space have been found even during fixation, suggesting a common mechanism for saccade and visual targeting (Moidell and Bedell, 1988; Schnier et al., 2010; Zimmer-

Received Nov. 22, 2010; Revised Feb. 23, 2011; accepted April 7, 2011.

Author contributions: E.Z. designed research; E.Z. performed research; E.Z. and M.L. analyzed data; E.Z. and M.L. wrote the paper.

The research was supported by the German Science Foundation (DFG LA-952/3), the German Federal Ministry of Education and Research project Visuo-spatial Cognition, and the EU Projects Eyeshots and "Space, Time and Numbers in the Brain" (FP7 ERC).

The authors declare no competing financial interests.

Correspondence should be addressed to Dr. Eckart Zimmermann, Department of Psychology, Università degli Studi di Firenze, 50135 Florence, Italy. E-mail: eckartzi@gmail.com.

DOI:10.1523/JNEUROSCI.6112-10.2011

Copyright $\odot 2011$ the authors $\quad 0270-6474 / 11 / 317341-08 \$ 15.00 / 0$ mann and Lappe, 2010). Modifications in saccade targeting must occur at multiple sites of the oculomotor pathways because different types of saccades (e.g., reactive or scanning saccades) show different adaptation behavior (Erkelens and Hulleman, 1993; Deubel, 1995a; Fujita et al., 2002; Collins and Doré-Mazars, 2006; Alahyane et al., 2007; Cotti et al., 2007; Zimmermann and Lappe, 2009). Reactive saccades are visually driven eye movements whose target localization is guided by visual input. Visual displacement vectors are principally sufficient to code target position in this case. Sequences of eye movements like scanning a visual scene can require a more complex coding of target positions. Changes in visual localization reflect the selective adaptability of different saccade types: The adaptation of scanning saccades induced mislocalization for flashed as well as for stationary objects. Reactive saccade adaptation, however, selectively affected flashed objects (Zimmermann and Lappe, 2009).

The reference frame of saccade adaptation so far has been tested only for reactive saccade adaptation. It was found that adaptation at eye positions around the straight-ahead direction transferred strongly to saccades starting at eccentric eye positions, suggesting a retino-centered coding of saccade adaptation in which the amount of adaptation depended only on the displacement vector of the eye (Semmlow et al., 1989; Frens and van Opstal, 1994; Deubel, 1995a; Albano, 1996; Noto et al., 1999; Havermann et al., 2010). However, inward adaptation at eccentric eye positions did not transfer completely to saccades starting from a central eye position, suggesting an eye position-dependent modulation for inward adaptation of reactive saccades (Havermann et al., 2010). 


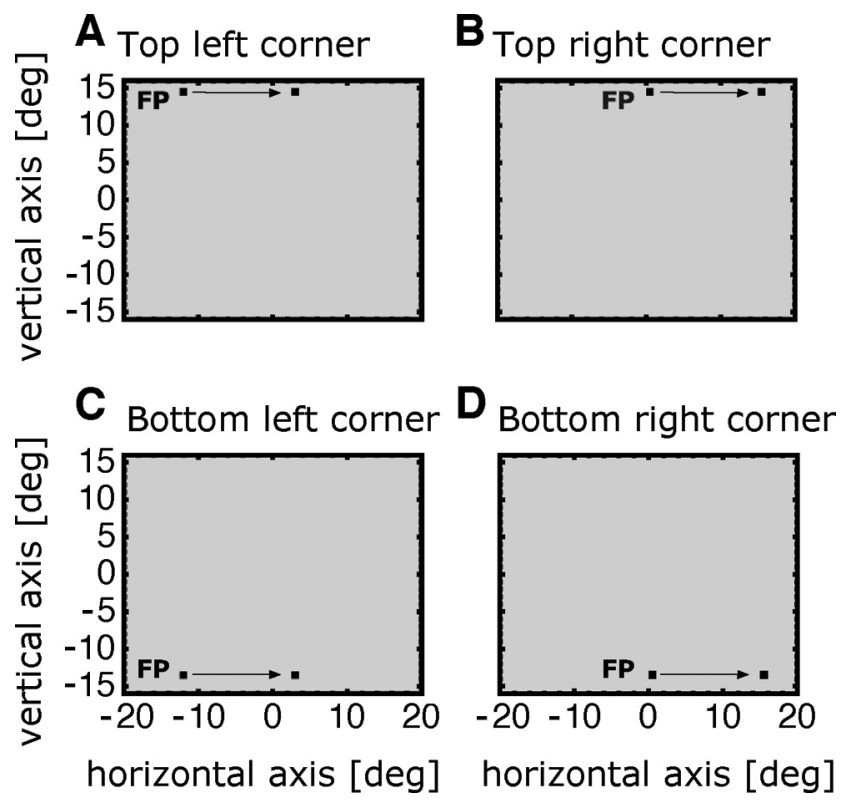

Figure 1. The experimental procedure used for examining the effect of eye position on reactive saccade amplitudes. $\boldsymbol{A}-\boldsymbol{D}$, Position of the fixation point (FP) and the saccade target in the four corners of the screen. The fixation point was shown for $1000 \mathrm{~ms}$. Simultaneously with the extinction of the fixation point, the saccade target appeared and the subject was required to perform a saccade. Then, the next fixation point appeared. In preadaptation and postadaptation trials, the positions at which the fixation points appeared were pseudorandomly selected. In adaptation trials, the fixation point always appeared in the bottom left corner and the saccade target appeared either rightward or upward. The arrow indicates the direction of the saccade and was not shown on the screen.

In the present study, we investigated the reference frame for outward adaptation of reactive and scanning saccades and the associated changes in visual localization. Consistent with earlier studies (Niemeier and Karnath, 2003; Zimmermann and Lappe, 2009), we expected that targeting of scanning saccade adaptation is coded in an extraretinal reference frame, which would predict reduced adaptation if the retinal position of the saccade target matches the adapted retinal position but the spatial position of the saccade target has changed. Changes in visual localization should show the same dependency on eye position.

\section{Materials and Methods}

To test the influence of eye position on saccade adaptation, we measured saccades with a fixed required amplitude size at different positions on the screen. Before adaptation, the combination of fixation point and saccade target was presented in every corner of the screen. In saccade adaptation trials, the targets were presented in only one of the four screen corners. This was the training location where adaptive motor learning was induced by an intrasaccadic target displacement. After 180 adaptation trials, targets were again shown in every corner of the screen. This method allowed us to determine the amount of adaptation transfer to saccade targets at different screen positions. Reactive and scanning saccades were studied in separate sessions. In each session, saccades were adapted in an outward direction and a localization task was included before and after adaptation. We chose adaptation in an outward direction because this condition induces strong changes in visual localization (Zimmermann and Lappe, 2010). To avoid visibility of the screen borders, we covered the display monitor with a transparent foil that reduced the luminance by $\sim 2 \log$ units.

Reactive saccades. A trial started with the presentation of a fixation point $\left(1 \times 1\right.$; luminance, $0.06 \mathrm{~cd} / \mathrm{m}^{2}$; red color $)$ on which the subject established and maintained fixation. Figure 1 shows the positions of the fixation point and the saccade target in each of the four corners. Fixation points were shown either in the bottom left corner of the screen $\left(12.5^{\circ}\right.$ to

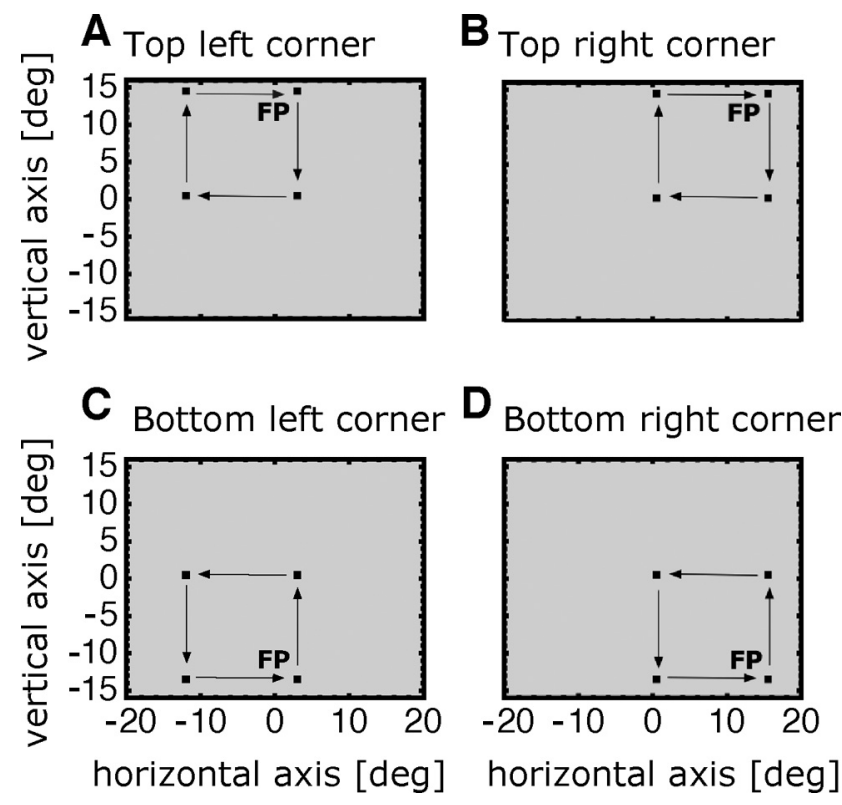

Figure 2. The experimental procedure used for examining the effect of eye position effects on scanning saccade amplitudes. $A-D$, Position of the scan path in the four corners of the screen. A trial started with the presentation of a fixation point (FP). When the subject pressed the space button on the keyboard, four targets were shown, which were permanently visible. When the scanning targets were presented in the top part of the screen, the subject had to scan the targets in a clockwise manner. When the targets were presented in the bottom part of the screen, the subject scanned the targets in a counterclockwise manner. Thus, the last saccade of each scan path was a rightward saccade for which adaptation was induced. When the execution of the last saccade was detected, all four saccade targets stepped $5^{\circ}$ in an outward direction. The arrow indicates the direction of the saccade and was not shown on the screen.

the left of and $14^{\circ}$ below the screen center) (Fig. $1 C$ ), in the bottom right corner of the screen (horizontal position at screen center and $14^{\circ}$ below the screen center) (Fig. $1 D$ ), in the top left screen corner $\left(12.5^{\circ}\right.$ to the left of and $14^{\circ}$ above the screen center) (Fig. $1 \mathrm{~A}$ ), or in the top right screen corner (horizontal position at screen center and $14^{\circ}$ above the screen center) (Fig. 1 B). After $1000 \mathrm{~ms}$, the fixation point was turned off and a saccade target appeared simultaneously. The saccade target always appeared $15^{\circ}$ to the right of the fixation point. After $1200 \mathrm{~ms}$, the saccade target was extinguished, and the next fixation point appeared in one of the four screen corners. In 55 preadaptation trials, saccades were performed 5 times in each of the three test screen corners and 15 times in the training location in a pseudorandom order. Trials 56-325 were adaptation trials. In these trials, the fixation point always appeared in the bottom left corner of the screen. In two-thirds of all adaptation trials, the saccade target was presented $15^{\circ}$ to the right of the fixation point. In one-third of the adaptation trials, the saccade target was presented $15^{\circ}$ above the fixation point to avoid subjects anticipating the next saccade target location. These trials were pseudorandomly intermixed with the other adaptation trials and were not used for analysis. Saccade adaptation was induced following the paradigm of McLaughlin (1967). Gaze position was measured online. When the eye tracker detected that the eye had traveled $2.5^{\circ}$ along the path of the saccade, the saccade target was displaced $5^{\circ}$ in the outward direction. In adaptation trials, the saccade target was displaced to the right of its initial position. In trials 326-415, saccades were again tested in all four corners of the screen. To determine the amount of saccade adaptation, we calculated the median over the saccade amplitudes from the pretrials and posttrials for each corner. Then, we subtracted the median postadaptation amplitude from the median preadaptation amplitude for each corner.

Scanning saccades. Scanning saccades were performed to four permanently visible targets. Scanning saccades were tested in the four corners of the screen (Fig. 2). The subject scanned the four targets $(1 \times 1$; luminance, $0.06 \mathrm{~cd} / \mathrm{m}^{2}$; red color) in clockwise order starting at the fixation point. The targets of the last saccade in the scan path in each corner were 
placed such that they matched the positions of the corresponding targets in the reactive saccade sessions. These saccades were used for analysis. The starting point of the last saccade of the scan path in the top left corner of Figure 2 was at a screen position of $15^{\circ}$ to the left of and $14^{\circ}$ above the screen center. At the same screen position, the fixation point in the reactive saccade sessions was presented as shown in the top left corner of Figure 2. The subject established fixation on the fixation point and to start the trial when ready by pressing the space button on the keyboard. Then, with the next frame, four saccade targets were visible. When gaze had arrived on the last target, the subject pressed the mouse button to end the trial. Then, the next fixation point appeared in one of the four screen corners. In 40 preadaptation trials, saccades were tested 10 times in each corner in a pseudorandom order. Trials $41-220$ were adaptation trials. In the adaptation trials, saccade targets always appeared in the bottom left corner of the screen. Saccade adaptation was induced when the last saccade of the scan path, a $15^{\circ}$ rightward saccade, was performed. When the eye tracker detected that the eye had traveled $2.5^{\circ}$ along the path of the saccade, the saccade target was displaced $5^{\circ}$ in the outward direction. In adaptation trials, the saccade target was displaced to the right of its initial position. In the last 90 trials, saccades were again tested in all four corners of the screen.

Localization procedure. A localization task was included in reactive saccade adaptation sessions and in scanning saccade adaptation sessions. Localization was tested five times in each corner. In a localization trial, the fixation point was presented in green color. Green fixation points signaled to the subjects to keep fixation during the whole trial and to avoid any eye movement. The fixation point was shown for $1000 \mathrm{~ms}$. After the fixation point was extinguished, the screen was blank for 100 $\mathrm{ms}$. Then a small bar $\left(0.3^{\circ} \times 4^{\circ}\right.$, luminance $\left.0.2 \mathrm{~cd} / \mathrm{m}^{2}\right)$ was flashed for 20 $\mathrm{ms}$. The bar was flashed $15^{\circ}$ to the right of the fixation point at the same position where the saccade target was presented in saccade trials. When the bar was turned off, the screen was blank again for $500 \mathrm{~ms}$. Then, a mouse pointer appeared, which the subject used to indicate the perceived position of the bar. The pointer appeared $1000 \mathrm{~ms}$ after the saccade near the bottom of the screen at a randomly assigned horizontal position between $35^{\circ}$ and $40^{\circ}$. Localization trials in which the eye tracker detected an eye movement were not used for analysis. The localization error was calculated as the deviation of the mouse click position from the position where the bar was presented. To determine the amount of mislocalization, we calculated the median over the localization errors from the pretrials and posttrials for each corner. Then, we subtracted the median postadaptation localization error from the median preadaptation localization error for each corner. When the fixation point was red, the subject performed a saccade.

Sequence of trials. In preadaptation sessions, saccade amplitudes and localization were tested in each corner five times. In addition to these trials, we measured 15 trials in the training location to obtain a stable baseline for the estimation of saccade adaptation. Thus, in total, 55 pretrials were measured. These were followed by 180 adaptation trials. Only in reactive saccade adaptation sessions were the adaptation trials pseudorandomly intermixed with 90 trials in which the saccade target was presented at another position to reduce predictability of the saccade target location. In 180 postadaptation trials, saccade amplitudes ( 10 trials in each corner) and localization (10 trials in each corner) were tested again. Also, 100 adaptation trials in the training location were included to avoid deadaptation induced by the intermixed test trials. The postadaptation trials were ordered in 20 blocks of nine trials each. One block consisted of three test trials, containing either the saccade or the localization task in one of the four screen corners pseudorandomly selected and six adaptation trials in the training location. Trials within one block were pseudorandomly ordered. To determine the amount of saccade adaptation, we calculated the median over the saccade amplitudes from the pretrials and posttrials for each corner. Then, we subtracted the median postadaptation amplitude from the median preadaptation amplitude for each corner.

Saccade latencies. We used latency differences as a test of whether we were successful in eliciting different saccade types. Saccade latencies in reactive saccade sessions were calculated as the time interval between onset of the saccade target and detection of the saccade. Since in the scanning saccade sessions, all saccade targets are visible from the trial start, saccade latency was calculated as the time interval between fixation onset on the fixation point and detection of the saccade. Latencies were in the normal reactive saccade range with $193.53 \pm 10.3$ ms averaged across subjects. Reactive saccade adaptation did not modify saccade latencies: Latencies of saccades before and after adaptation were almost identical (before adaptation: $192.64 \pm 10.19 \mathrm{~ms}$; after adaptation: $194.42 \pm 10.41$ $\mathrm{ms})$. Scanning saccade latencies were much higher with $444.22 \pm 72.35$ ms averaged across subjects. Saccade latencies were somewhat smaller after adaptation (before adaptation: $462.84 \pm 80.79 \mathrm{~ms}$; after adaptation: $425.60 \pm 63.90 \mathrm{~ms})$. To test whether reactive and scanning saccade latencies were significantly different, we conducted a two-way repeatedmeasures ANOVA with saccade type (reactive/scanning) and adaptation state (preadaptation/postadaptation) as factors. A significant main effect of saccade type $(F=15.3, p=0.04)$ confirmed that we were successful in eliciting different saccade types in the different conditions.

Participants. Nine subjects (two male, seven female; one author, eight naive subjects; mean age $=23$ years) participated in all of the experiments. All subjects were students from the psychology department of the University Münster and had normal or corrected-to-normal vision. Subjects gave informed consent. All subjects underwent all experimental conditions. The experiments were performed along the principles in the Declaration of Helsinki.

\section{Results}

The influence of eye position on the magnitude of reactive and scanning saccade adaptation and on adaptation-induced mislocalization was tested. All sessions were run in complete darkness to avoid localization being influenced by visual landmarks. Visual localization was tested after adaptation of reactive and scanning saccades. Both localization and saccade amplitudes were tested in each session to compare the effects of eye position. Each subject performed each session two to four times until sufficient data for statistical analysis were collected. Data from these sessions were pooled for each subject. Gain values for saccade amplitudes were obtained by first calculating the median saccade amplitude of the preadaptation trials separately for saccade amplitude data from all four screen corners. Then, all saccade amplitude data from all trials in every corner of the screen were divided by the specific median saccade amplitude. Similarly, all visual localization data were divided by the specific median localization. In the following, single-subject median values are reported with the lower and upper quartiles. We calculated mean adaptation and mean mislocalization when averaging across subjects. These mean values were tested for normal distribution with a Kolmogorov-Smirnov test before calculating the ANOVA.

\section{Eye position effects on reactive saccade adaptation and mislocalization}

Figure 3 shows saccade amplitudes over a single session of reactive saccade adaptation in the outward direction. The saccade adaptation training location was in the bottom left corner of the screen (the according saccade adaptation curve is shown in Fig. $3 C$ ). In 55 preadaptation trials, saccades were tested in all four corners of the screen in pseudorandom order. Median saccade gain in the preadaptation trials of saccades performed in the training location of the bottom left corner was by definition $100 \%(96.5 \% ; 104.3 \%)$ for this subject (Fig. 3C). Localization of bars briefly flashed at $15^{\circ}$ to the right of the fixation point while the subject fixated was also tested in all four corners of the screen. In the training location, the localization gain for the subject in Figure 3 was $100 \%$ (96.5\%; 103.2\%).

From trial 56, saccade adaptation was induced with an intrasaccadic target displacement of $5^{\circ}$. The target was displaced in an outward direction (i.e., to the right of its initial position). Trials 
326-415 were postadaptation trials. In the postadaptation trials, saccades were tested in all four corners of the screen, as in the preadaptation trials. Median saccade gain in the postadaptation trials of saccades performed in the training location was $114 \%(109.3 \% ; 117.4 \%)$ for this subject (Fig. 3C).

To determine the effect of eye position on saccade adaptation, we compared the amount of adaptation in the training location with the amount of adaptation in the remaining corners. Adaptation magnitude of saccades performed in positions distant from the training location was reduced: In the bottom right corner (Fig. 3D), the saccade gain was unchanged (100\%: 99\%; $102.4 \%$ ); in the top left corner (Fig. 3A), it changed to $106 \%(104.5 \% ; 111.9 \%)$; and in the top right corner (Fig. $3 B$ ), it changed to $106 \%(104.2 \% ; 108.3 \%)$. Similarly to the saccade amplitudes, localization gain was shifted in the direction of adaptation. In the training location (Fig. 3C), localization gain changed to $109 \%$ (103.6\%; 112.9\%).

Localization gain change differed at other positions on the screen: In the bottom right corner (Fig. 3D), the gain reduced to $107 \%(99.8 \% ; 110.9 \%)$; in the top left corner (Fig. $3 A$ ), it increased to $111 \%(108.1 \% ; 113.4 \%)$; and in the top right corner (Fig. 3B), it reduced to $104 \%$ (102.6\%; 106.6\%).

The amount of reactive saccade outward adaptation averaged over all subjects is shown in Figure 4. The diameter of each inner circle (in black) represents the mean adaptation magnitude in the corresponding corner of the screen. The difference between the diameter of the outer circles and the diameter of the inner circle reflects the size of the SE. Magnitude of reactive saccade adaptation is shown in Figure $4 \mathrm{~A}$. Average saccade gain change in the training location in the bottom left corner was $109 \pm 1 \%$. Adaptation magnitude of saccades tested at other screen positions was reduced, as can be seen by the smaller size of the circles. In the bottom right screen corner, mean saccade gain change was $103 \pm 1 \%$; in the top left corner, it was $107 \pm 2 \%$; and in the top right corner, it was $107 \pm 1 \%$. Subtracting the median postadaptation saccade amplitude by the median preadaptation saccade amplitude gave the following amounts of adaptation: $1.47 \pm 0.2^{\circ}$ for the bottom left corner, $0.6 \pm 0.15^{\circ}$ for the bottom right corner, $1.11 \pm 0.24^{\circ}$ for the top left corner, and $0.7 \pm 0.27^{\circ}$ for the top right corner. A one-way ANOVA confirmed a significant eye position effect for reactive saccade outward adaptation $(F=4.97$, $\mathrm{df}=3 ; p=0.009)$.

The magnitude of adaptation-induced mislocalization is shown in Figure $4 B$. Average mislocalization gain change in the training location in the bottom left corner was $104 \pm 1 \%$. Mislocalization in the remaining screen positions was reduced similarly to the decrease of saccade adaptation magnitude at other screen positions. In the bottom right screen corner, mean mislocalization gain change was $104 \pm 1 \%$; in the top left corner, it was $104 \pm 3 \%$; and in the top right corner, it was $105 \pm 1 \%$. Subtracting the median postadaptation localization amplitude by the me-

\section{Reactive saccades}
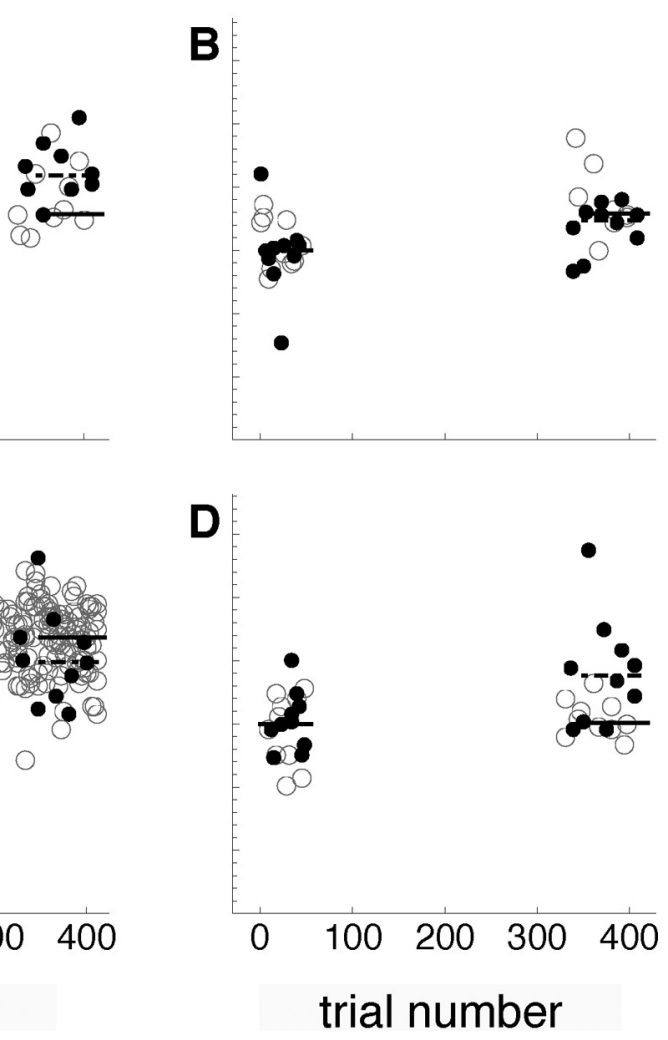

trial number
Figure 3. Example saccade amplitudes and localization error from a session in which reactive saccades were adapted. The saccade adaptation training location was in the bottom left corner of the screen. $\boldsymbol{A}, \boldsymbol{B}, \boldsymbol{D}$, Preadaptation and postadaptation postadaptation visual localization. C, Adaptation curve for reactive saccades performed in the training location (open dots) and preadaptation and postadaptation visual localization (filled dots). The black solid lines indicate the median saccade amplitude and the black dashed lines indicate the median localization error of preadaptation and postadaptation trials.

\section{Reactive saccades}

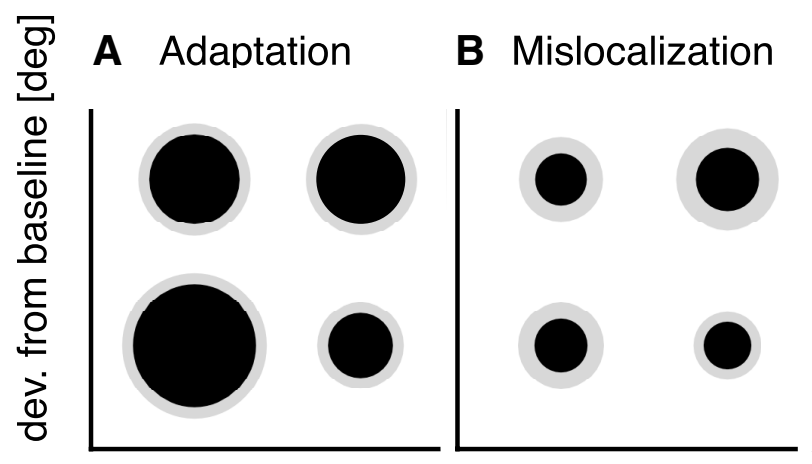

Figure 4. A, Magnitude of reactive saccade adaptation at the four eccentricities averaged over all subjects. $\boldsymbol{B}$, Magnitude of reactive saccade adaptation-induced mislocalization at the four eccentricities averaged over all subjects. The diameter of each inner circle represents the adaptation magnitude at the corresponding screen position. The difference between the diameter of the outer circles and the diameter of the inner circles represents the SE of the sample mean at each corresponding screen position. Adaptation magnitude was calculated as the difference between median amplitude sizes from preadaptation and postadaptation trials.

dian preadaptation localization amplitude gave the following amounts of mislocalization: $0.63 \pm 0.39^{\circ}$ for the bottom left corner, $0.57 \pm 0.26^{\circ}$ for the bottom right corner, $0.61 \pm 0.39^{\circ}$ for the top left corner, and $0.77 \pm 0.47^{\circ}$ for the top right corner. Mislocalization induced by reactive saccade outward adaptation did 


\section{Scanning saccades}

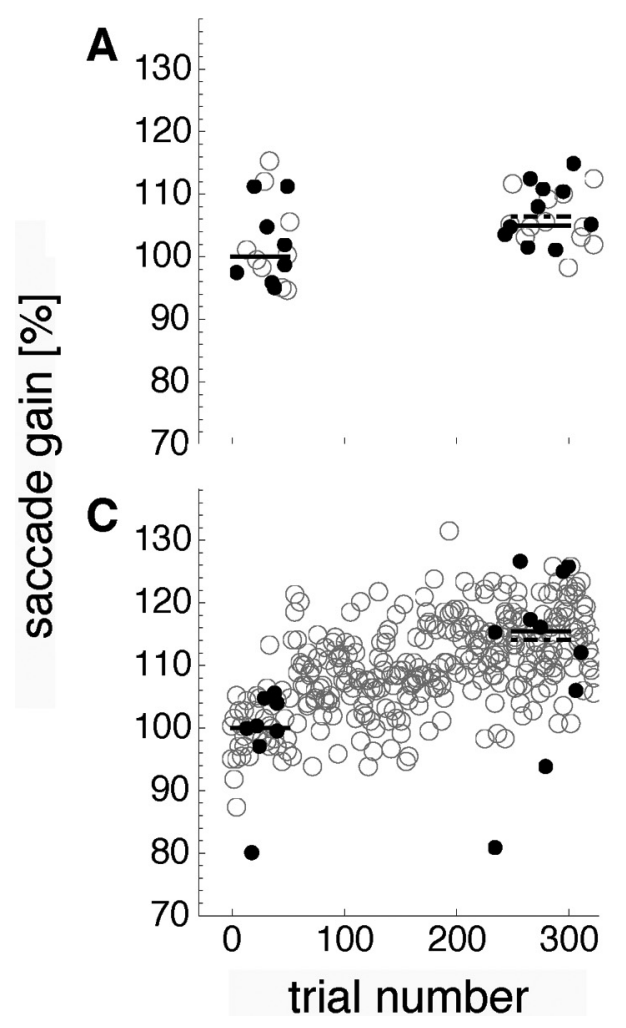

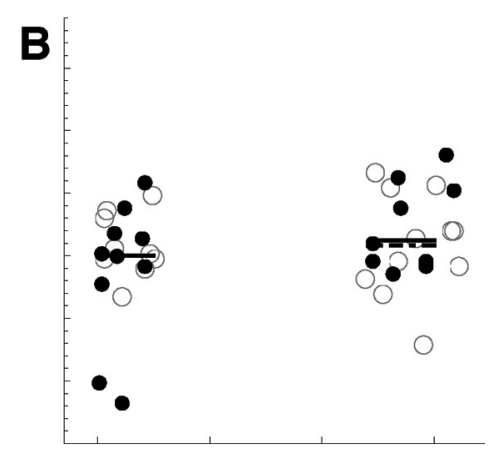

D

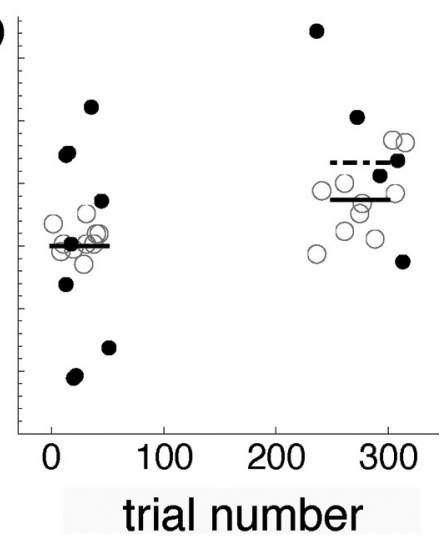

Figure 5. Example saccade amplitudes and localization from a session in which scanning saccades were adapted. Data derive from the same subject as in Figure 3. The saccade adaptation training location was in the bottom left corner of the screen. $A, B, D$, Preadaptation and postadaptation scanning saccade amplitudes tested at the corresponding screen locations (open dots). Filled dots indicate preadaptation and postadaptation visual localization. $\boldsymbol{C}$, Adaptation curve for scanning saccades performed in the training location (open dots) and preadaptation and postadaptation visual localization (filled dots). The black solid lines indicate the median saccade amplitude and the black dashed lines indicate the median localization error of preadaptation and postadaptation trials.

not change significantly at different eye positions, as revealed by a one-way repeated-measures ANOVA $(F=0.842, \mathrm{df}=3$; $p=0.482$ ).

\section{Eye position effects on scanning saccade adaptation and mislocalization}

Saccade amplitudes over a single session of scanning saccade adaptation in outward direction are shown in Figure 5. Data are from the same subject as in Figure 3. The saccade adaptation training location was the bottom left screen corner (the according saccade adaptation curve is shown in Fig. 5C). The first 40 trials were preadaptation trials. Scanning saccades were tested in all four positions on the screen in pseudorandom order. The last saccade of each scan path was analyzed. Median saccade gain in the preadaptation trials of saccades performed in the training location of the bottom left corner (Fig. 5 C) had a gain of $100 \%$ $(94.5 \% ; 111.7 \%$; gray points) for this subject. Intermixed with the saccade trials were trials in which the subject localized bars that were briefly flashed $15^{\circ}$ to the right of the fixation point. The localization gain for the subject in Figure 5 was 100\% (96.7\%; $103.6 \%$; black points). From trial 41 onward, the saccade target in the top right corner was displaced $5^{\circ}$ in the outward direction to the right of its initial position. Trials 220-325 were postadaptation trials in which saccade gain was tested in all four corners of the screen. In the postadaptation trials, median saccade gain in the training location (Fig. $5 C$ ) was $114 \%(109.5 \pm 117.6 \%)$. The increase of saccade gain was smaller at other positions: In the bottom right corner (Fig. 5D), it was 107\% (102.3 \pm $109.7 \%)$; in the top left corner (Fig. $5 A$ ), it was $105 \%(102.8 \pm 108.9 \%)$; and in the top right corner (Fig. $5 B$ ), it was $102 \%(96.2 \pm 110.6 \%)$. In addition, localization gain was shifted in the direction of adaptation. In the training location (Fig. 5C), localization gain was $115 \%(105.9 \% ; 124.7 \%)$. Similarly to the saccade gain, this shift was smaller at other positions on the screen: In the bottom right corner (Fig. 5D), it was 113\% (110.9\%; $120.6 \%$ ); in the top left corner (Fig. $5 A$ ), it was $106 \%(103.5 \% ; 110.5 \%)$; and in the top right corner (Fig. 5B), it was 102\% (99.1\%; $110.1 \%)$.

The amount of scanning saccade outward adaptation averaged over all subjects is shown in Figure 6. Results from scanning saccade adaptation sessions are shown in Figure 6 A. Average adaptive saccade gain change in the training location in the bottom left corner was $110 \pm 1 \%$. Mean gain change of saccades tested at other screen positions was reduced, as can be seen by the smaller size of the circles. In the bottom right screen corner, mean saccade gain change was $107 \pm 1 \%$; in the top left corner, it was $105 \pm 1 \%$; and in the top right corner, it was $102 \pm 2 \%$. Subtracting the median postadaptation saccade amplitude by the median preadaptation saccade amplitude gave the following amounts of adaptation: $1.54 \pm 0.09^{\circ}$ for the bottom left corner, $1.11 \pm 0.15^{\circ}$ for the bottom right corner, $0.98 \pm 0.23^{\circ}$ for the top left corner, and $0.39 \pm 0.25^{\circ}$ for the top right corner. Significant eye position effects on scanning saccade adaptation were confirmed by a one-way repeated-measures ANOVA $(F=5.151, \mathrm{df}=3 ; p=0.007)$. A very similar result was observed for mislocalization after adaptation of scanning saccades, which is shown in Figure $6 \mathrm{~B}$. Average gain change of mislocalization was highest in the training location (109 \pm $2 \%$ ) and declined at the other screen positions: In the bottom right screen corner, mean mislocalization gain change was $106 \pm 2 \%$; in the top left corner, it was $103 \pm 2 \%$; and in the top right corner, it was $101 \pm 1 \%$. Subtracting the median postadaptation localization amplitude by the median preadaptation localization amplitude gave the following amounts of mislocalization: $1.2 \pm 0.27^{\circ}$ for the bottom left corner, $0.82 \pm 0.25^{\circ}$ for the bottom right corner, $0.33 \pm 0.22^{\circ}$ for the top left corner, and $0.21 \pm 0.25^{\circ}$ for the top right corner. For mislocalization induced by scanning saccade outward adaptation, significant eye position effects were confirmed by a one-way repeatedmeasures ANOVA $(F=4.294, \mathrm{df}=3 ; p=0.016)$.

To compare eye position modulation on reactive and scanning saccade adaptation, we calculated a two-way repeated-measures ANOVA with saccade type (reactive/scanning) and screen positions (all four corners) as factors. A significant main effect for screen positions $(F=5.053 \mathrm{df}=3 ; p=0.07)$ confirmed eye position effects on saccade adaptation. Eye position modulated scanning saccade outward adaptation stronger than reactive saccade outward adaptation, as revealed by a significant interaction effect $(F=4.53, \mathrm{df}=1$; 


\section{Scanning saccades}

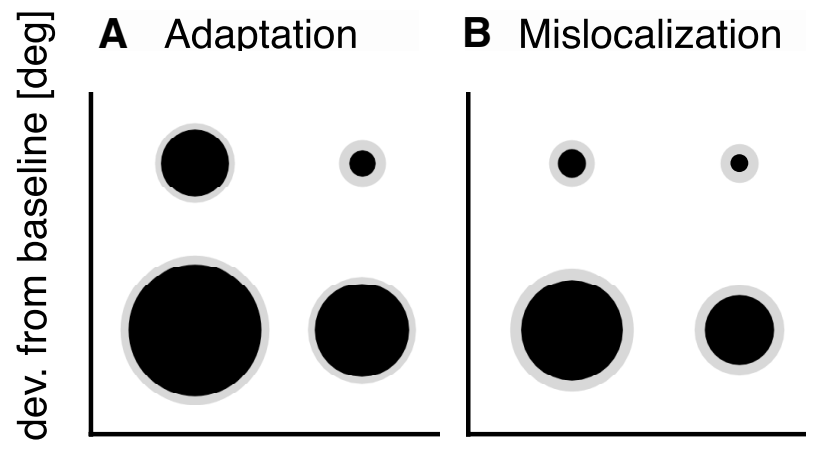

Figure 6. $\quad A$, Magnitude of scanning saccade adaptation at the four eccentricities averaged over all subjects. $\boldsymbol{B}$, Magnitude of scanning saccade adaptation-induced mislocalization at the four eccentricities averaged over all subjects. The same conventions are used as in Figure 4.

$p=0.02)$. For mislocalization magnitude, a two-way repeatedmeasures ANOVA was conducted with saccade type (reactive/scanning) and screen positions (all four corners) as factors. Also for mislocalization, an effect of eye position was revealed by the significant main effect of screen position $(F=5.199, \mathrm{df}=3 ; p=0.008)$.

\section{Discussion}

Orbital eye position modulated the magnitude of reactive and scanning saccade adaptation and the associated mislocalization. These results demonstrate that saccade adaptation is not coded in purely retinal coordinates. If saccade adaptation relies on a retinotopic reference frame, adaptation magnitude should remain constant at all tested orbital positions. But this clearly was not the case: The amount of reactive saccade adaptation was reduced when saccades were adapted at one eye position and then tested at another. Much more so, the results from scanning saccade adaptation reveal a clear involvement of an extraretinal reference frame. Adaptation magnitude decreased strongly at different eye positions. This effect was most pronounced when horizontal and vertical eye positions were simultaneously changed. The reduction of adaptation magnitude for scanning saccades was significantly stronger than for reactive saccades. This is consistent with earlier studies that found selective adaptability of reactive and scanning saccades (Deubel, 1995b; Collins and Doré-Mazars, 2006; Zimmermann and Lappe, 2009) and a stronger influence of scanning saccade adaptation on visual localization (Collins and Doré-Mazars, 2006; Cotti et al., 2007; Zimmermann and Lappe, 2009). For scanning saccades, eye position effects were found for adaptation and for mislocalization. For reactive saccade adaptation, however, eye position modulated adaptation but not mislocalization. Eye position effects for reactive saccades were weaker than for scanning saccades (as confirmed by the two-way ANOVA); thus, it is very likely that they were not sufficiently strong to induce significant effects for mislocalization. The reason adaptation but not mislocalization showed significant effects is probably the somewhat higher scatter for localization than for adaptation (Fig. 4). Because localization was tested during ocular fixation, mislocalization phenomena that occur briefly before and during execution of a saccade (Ross et al., 2001; Melcher and Colby, 2008) are not related to the present results. Also, the modulation of visual mislocalization magnitude by orbital eye position was very similar to the modulation of saccade adaptation by orbital eye position. This result reinforces earlier evidence for a common mechanism of saccade and visual targeting (Collins et al., 2007; Zimmermann and Lappe, 2009, 2010). If visual localization relies on motor metrics, any changes in motor metrics should be paralleled by homogeneous localization changes in visual perception. This common mechanism must be in a structure that receives signals representing eye position.

Which role might eye position play in localization? In the absence of visual landmarks, the visual system needs a position code to assess and maintain the locations of objects in space. A computationally efficient strategy is to use oculomotor metrics (i.e., the vectors of those saccades that would be performed to the same object) as code for spatial position. A visual position code uninformed of motor metrics would run into the problem of misalignment, resulting eventually in inaccurate motor behavior. Our data, however, demonstrate that when motor metrics are artificially changed, visual perception is instantaneously modified according to the new motor behavior. A neural implementation might consist of a connection of a motor map that codes eye position with a visual map coding visual objects' features. In this way, visual features would be structured by eye positions that were required to bring the spatially corresponding visual features into the fovea. If the metrics of the motor map experience modulations at eccentric eye positions, these changes must necessarily be reflected in the spatial perception of the visual objects. Indeed, in our data, changes in visual localization were modulated by eye position similarly to modulations in saccade adaptation magnitude.

Where in the sensorimotor transformation can such a connection between visual and motor maps occur? Eye position gain field modulations of visual signals have been found in the parietal cortex (Andersen and Mountcastle, 1983; Andersen et al., 1985; Galletti et al., 1993; Brotchie et al., 1995; Bremmer et al., 1998; Snyder et al., 1998), the premotor cortex (Schlag et al., 1992; Graziano et al., 1997; Mushiake et al., 1997; Graziano and Gross, 1998), and the frontal eye field (Balan and Ferrera, 2003; Cassanello and Ferrera, 2007). These structures can principally deliver information about eye position. The neural locus of saccade adaptation is not fully determined. In humans, differences in adaptation between saccade types (Deubel, 1995a; Alahyane et al., 2007; Cotti et al., 2007; Zimmermann and Lappe, 2009) and also between adaptation directions (Ethier et al., 2008; Panouillères et al., 2009; Zimmermann and Lappe, 2010) suggest that oculomotor plasticity can occur at multiple stages of the oculomotor system. Electrophysiological studies in monkeys, however, have found changes after saccade adaptation only in the oculomotor vermis of the cerebellum (Catz et al., 2008) and lower brainstem structures. The cerebellum receives input from saccadetargeting structures such as the superior colliculus and the frontal eye field. Eye position information could be mediated via these structures and induce gaze-dependent adaptation in the cerebellum. Eye position signals have been found in different stages of the oculomotor system: Saccadic dysmetria after cerebellar lesion and inactivation are dependent on eye position (Ritchie, 1976; Goldberg et al., 1993; Robinson et al., 1993). Ascending projections from the cerebellum to cortical areas might be relevant for changes in visual and motor localization. Indeed, saccade adaptation was reduced in patients with lesions in the cerebral thalamus (Gaymard et al., 2001). Cerebellar projections to the parietal cortex via cerebellothalamocortical pathways have been shown to be functionally relevant for the adaptive control of eye movements as well as for adaptation of visual localization (Prevosto et al., 2010). Our results leave open the possibility that the adaptation and mislocalization effects are functionally spatiotopic. In our experiments, we manipulated eye position, but the saccade 
targets were presented at the same retinal position. Nonretinotopic eye position encoding in spatiotopic coordinates has been reported in single-cell recording studies for areas V6 and VIP (Galletti et al., 1993; Bremmer et al., 1998). Functional spatiotopy does not necessarily require neurons to encode information in spatiotopic coordinates. Retinal encoding plus gaze modulation would also lead to functional spatiotopy. Spatiotopic encoding in human visual cortex is a matter of debate (Melcher and Morrone, 2003; d'Avossa et al., 2007; Gardner et al., 2008; Morris et al., 2010; Pertzov et al., 2010, 2011; Burr and Morrone, 2011). It is an interesting question whether the nonretinotopic adaptation and the accompanied mislocalization are coded in spatiotopic coordinates.

Other explanations than motor signals as position code for visual perception are possible: First, the intrasaccadic target displacement could induce changes separately in visual and motor maps. To explain the similar modulation of visual and saccade targeting by eye position, one would have to assume that gain field neurons induce simultaneously comparable distortions in the metrics of visual and motor maps. A further alternative is that the effect of adaptation is purely visual and takes place solely in visual structures, and that the adaptive changes in saccade amplitude are a result of this visual adaptation. It is not unreasonable that postsaccadic errors recalibrate visual maps to maintain visual stability. One would have to assume then that eye position signals modulate visual areas. This hypothesis, however, is quite speculative and is in contrast to the well documented changes in motor areas following saccade adaptation (Hopp and Fuchs, 2004; Pélisson et al., 2010).

Several earlier studies measured eye position effects on reactive saccade adaptation. A similar conclusion to ours was reached by Semmlow et al. (1989), who adaptively increased saccade vectors in one direction and tested saccades of many different sizes and initial starting positions in the same direction. However, since the tested saccades were performed in the same direction, this method did not allow differentiation between a craniotopic coding of target position and the retinal spread of adaptation to saccades of differing sizes. Earlier studies that investigated inward adaptation did not find eye position effects (Frens and van Opstal, 1994; Deubel, 1995a). Albano (1996) tested eye position dependency of reactive saccade adaptation in inward and outward adaptation at a very small range. Adaptation magnitude was reduced at some positions and not at others. None of these studies, however, tested scanning saccades, which is the condition in which we found the strongest effects. Consistent with our results, however, other studies have demonstrated that it is possible to adapt saccades with fixed vectors in inward and outward directions simultaneously if only the initial eye position is changed (Shelhamer and Clendaniel, 2002; Alahyane and Pélisson, 2004; Tian and Zee, 2010). Since in a purely retinotopic coding scheme, simultaneous inward and outward adaptation should cancel each other, these studies highlighted the use of eye position signals in oculomotor plasticity.

We conclude that saccade targeting and visual localization rely on a common position code that is modulated by eye position. This extraretinal reference frame provides the coordinates for oculomotor plasticity and for the associated metrics of visual localization. A shared position code for saccade eye movements and visual space perception ensures that modifications in oculomotor parameters are reflected in visual localization, thereby avoiding the problem of misalignment between perceptual and motor space.

\section{References}

Alahyane N, Pélisson D (2004) Eye position specificity of saccadic adaptation. Invest Ophthalmol Vis Sci 45:123-130.

Alahyane N, Salemme R, Urquizar C, Cotti J, Guillaume A, Vercher JL, Pélisson D (2007) Oculomotor plasticity: are mechanisms of adaptation for reactive and voluntary saccades separate? Brain Res 1135:107-121.

Albano JE (1996) Adaptive changes in saccade amplitude: oculocentric or orbitocentric mapping? Vision Res 36:2087-2098.

Andersen RA, Mountcastle VB (1983) The influence of the angle of gaze upon the excitability of the light-sensitive neurons of the posterior parietal cortex. J Neurosci 3:532-548.

Andersen RA, Essick GK, Siegel RM (1985) Encoding of spatial location by posterior parietal neurons. Science 230:456-458.

Awater H, Burr D, Lappe M, Morrone MC, Goldberg ME (2005) Effect of saccadic adaptation on localization of visual targets. J Neurophysiol 93:3605-3614.

Bahcall DO, Kowler E (1999) Illusory shifts in visual direction accompany adaptation of saccadic eye movements. Nature 400:864-866.

Balan PF, Ferrera VP (2003) Effects of gaze shifts on maintenance of spatial memory in macaque frontal eye field. J Neurosci 23:5446-5454.

Bremmer F, Pouget A, Hoffmann KP (1998) Eye position encoding in the macaque posterior parietal cortex. Eur J Neurosci 10:153-160.

Brotchie PR, Andersen RA, Snyder LH, Goodman SJ (1995) Head position signals used by parietal neurons to encode locations of visual stimuli. Nature 375:232-235.

Bruno A, Morrone MC (2007) Influence of saccadic adaptation on spatial localization: comparison of verbal and pointing reports. J Vis 7:16.1-16.13.

Burr DC, Morrone MC (2011) Spatiotopic coding and remapping in humans. Philos Trans R Soc Lond B Biol Sci 366: 504-515.

Cassanello CR, Ferrera VP (2007) Computing vector differences using a gain field-like mechanism in monkey frontal eye field. J Physiol 582:647-664.

Catz N, Dicke PW, Thier P (2008) Cerebellar-dependent motor learning is based on pruning a Purkinje cell population response. Proc Natl Acad Sci U S A 105:7309-7314.

Collins T, Doré-Mazars K (2006) Eye movement signals influence perception: evidence from the adaptation of reactive and volitional saccades. Vision Res 46:3659-3673.

Collins T, Doré-Mazars K, Lappe M (2007) Motor space structures perceptual space: evidence from human saccadic adaptation. Brain Res 1172:32-39.

Cotti J, Guillaume A, Alahyane N, Pelisson D, Vercher JL (2007) Adaptation of voluntary saccades, but not of reactive saccades, transfers to hand pointing movements. J Neurophysiol 98:602-612.

d'Avossa G, Tosetti M, Crespi S, Biagi L, Burr DC, Morrone MC (2007) Spatiotopic selectivity of BOLD responses to visual motion in human area MT. Nat Neurosci 10:249-255.

Deubel H (1995a) Is adaptation saccadic context-specific? In: Eye movement research: mechanisms, processes and applications, Vol 6, Chap 16 (Groner R, d'Ydewalle G, eds), pp 177-187. Amsterdam: Elsevier.

Deubel H (1995b) Separate adaptive mechanisms for the control of reactive and volitional saccadic eye movements. Vision Res 35:3529-3540.

Erkelens CJ, Hulleman J (1993) Selective adaptation of internally triggered saccades made to visual targets. Exp Brain Res 93:157-164.

Ethier V, Zee DS, Shadmehr R (2008) Changes in control of saccades during gain adaptation. J Neurosci 28:13929-13937.

Frens MA, van Opstal AJ (1994) Transfer of short-term adaptation in human saccadic eye movements. Exp Brain Res 100:293-306.

Fujita M, Amagai A, Minakawa F, Aoki M (2002) Selective and delay adaptation of human saccades. Brain Res Cogn Brain Res 13:41-52.

Galletti C, Battaglini PP, Fattori P (1993) Parietal neurons encoding spatial locations in craniotopic coordinates. Exp Brain Res 96:221-229.

Gardner JL, Merriam EP, Movshon JA, Heeger DJ (2008) Maps of visual space in human occipital cortex are retinotopic, not spatiotopic. J Neurosci 28:3988-3999.

Gaymard B, Rivaud-Péchoux S, Yelnik J, Pidoux B, Ploner CJ (2001) Involvement of the cerebellar thalamus in human saccade adaptation. Eur J Neurosci 14:554-560.

Goldberg M, Musil S, Fitzgibbon E, Smith M, Olson C (1993) The role of the cerebellum in the control of saccadic eye movements. In: Role of the 
cerebellum and basal ganglia in voluntary movements (Mano N, Hamada I, DeLong MR, eds), pp 203-211. Amsterdam: Elsevier.

Graziano MS, Gross CG (1998) Visual responses with and without fixation: neurons in premotor cortex encode spatial locations independently of eye position. Exp Brain Res 118:373-380.

Graziano MS, Hu XT, Gross CG (1997) Visuospatial properties of ventral premotor cortex. J Neurophysiol 77:2268-2292.

Havermann K, Zimmermann E, Fattori P, Lappe M (2010) Eye position effects in the adaptation of reactive saccades. Soc Neurosci Abstr 36:532.2.

Hopp JJ, Fuchs AF (2004) The characteristics and neuronal substrate of saccadic eye movement plasticity. Prog Neurobiol 72:27-53.

McLaughlin SC (1967) Parametric adjustment in saccadic eye movements. Percept Psychophys 2:359-362.

Melcher D, Colby CL (2008) Trans-saccadic perception. Trends Cogn Sci 12:466-473.

Melcher D, Morrone MC (2003) Spatiotopic temporal integration of visual motion across saccadic eye movements. Nat Neurosci 6:877-881.

Moidell BG, Bedell HE (1988) Changes in oculocentric visual direction induced by the recalibration of saccades. Vision Res 28:329-336.

Morris AP, Liu CC, Cropper SJ, Forte JD, Krekelberg B, Mattingley JB (2010) Summation of visual motion across eye movements reflects a nonspatial decision mechanism. J Neurosci 30:9821-9830.

Mushiake H, Tanatsugu Y, Tanji J (1997) Neuronal activity in the ventral part of premotor cortex during target-reach movement is modulated by direction of gaze. J Neurophysiol 78:567-571.

Niemeier M, Karnath HO (2003) Stimulus-driven and voluntary saccades are coded in different coordinate systems. Curr Biol 13:585-589.

Noto CT, Watanabe S, Fuchs AF (1999) Characteristics of simian adaptation fields produced by behavioral changes in saccade size and direction. J Neurophysiol 81:2798-2813.

Panouillères M, Weiss T, Urquizar C, Salemme R, Munoz DP, Pélisson D (2009) Behavioral evidence of separate adaptation mechanisms controlling saccade amplitude lengthening and shortening. J Neurophysiol 101:1550-1559.

Pélisson D, Alahyane N, Panouillères M, Tilikete C (2010) Sensorimotor adaptation of saccadic eye movements. Neurosci Biobehav Rev 34:1103-1120.
Pertzov Y, Zohary E, Avidan G (2010) Rapid formation of spatiotopic representations as revealed by inhibition of return. J Neurosci 30:8882-8887.

Pertzov Y, Avidan G, Zohary E (2011) Multiple reference frames for saccadic planning in the human parietal cortex. J Neurosci 31:1059-1068.

Prevosto V, Graf W, Ugolini G (2010) Cerebellar inputs to intraparietal cortex areas LIP and MIP: functional frameworks for adaptive control of eye movements, reaching, and arm/eye/head movement coordination. Cereb Cortex 20:214-228.

Ritchie L (1976) Effects of cerebellar lesions on saccadic eye movements. J Neurophysiol 39:1246-1256.

Robinson FR, Straube A, Fuchs AF (1993) Role of the caudal fastigial nucleus in saccade generation. II. Effects of muscimol inactivation. J Neurophysiol 70:1741-1758.

Ross J, Morrone MC, Goldberg ME, Burr DC (2001) Changes in visual perception at the time of saccades. Trends Neurosci 24:113-121.

Schlag J, Schlag-Rey M, Pigarev I (1992) Supplementary eye field: influence of eye position on neural signals of fixation. Exp Brain Res 90:302-306.

Schnier F, Zimmermann E, Lappe M (2010) Adaptation and mislocalization fields for saccadic outward adaptation in humans. J Eye Mov Res 3:4.1-4.18.

Semmlow JL, Gauthier GM, Vercher JL (1989) Mechanisms of short-term saccadic adaptation. J Exp Psychol Hum Percept Perform 15:249-258.

Shelhamer M, Clendaniel R (2002) Sensory, motor, and combined contexts for context-specific adaptation of saccade gain in humans. Neurosci Lett 332:200-204.

Snyder LH, Grieve KL, Brotchie P, Andersen RA (1998) Separate body- and world-referenced representations of visual space in parietal cortex. Nature 394:887-891.

Tian J, Zee DS (2010) Context-specific saccadic adaptation in monkeys. Vision Res 50:2403-2410.

Zimmermann E, Lappe M (2009) Mislocalization of flashed and stationary visual stimuli after adaptation of reactive and scanning saccades. J Neurosci 29:11055-11064.

Zimmermann E, Lappe M (2010) Motor signals in visual localization. J Vis $10: 2.1-2.11$ 